\title{
Investigation of Resistance and Clonal Relatedness Among Nosocomial Acinetobacter Isolates
}

\author{
Ahmet Çalışkan ${ }^{1}$, Rıza Durmaz ${ }^{2}$, Canan Ateş Gürsoy ${ }^{3}$, Nilay Ildız $^{4}$ \\ ${ }^{1}$ Department of Medical Microbiology, Faculty of Medicine, Pamukkale University, Denizli, Turkey \\ ${ }^{2}$ Department of Medical Microbiology, Faculty of Medicine, Y1ldırım Beyazıt University, Ankara, Turkey \\ ${ }^{3}$ Department of Medical Microbiology, Faculty of Medicine, Inonu University, Malatya, Turkey \\ ${ }^{4}$ Department of Pharmaceutical Microbiology, Faculty of Pharmacy, Erciyes University, Kayseri, Turkey
}

Received: 24 June 2019, Accepted: 29 July2019, Published online: 28 August 2019 (C) Ordu University Institute of Health Sciences, Turkey, 2019

\begin{abstract}
Objective: This study focused on Acinetobacter spp. that were isolated from inpatients to determine the resistance rates to antibiotics and to monitor the resistance increase over the years.

Method: To determine whether there was a clonal relationship between the strains using pulsed field gel electrophoresis (PFGE), to evaluate the epidemiological and clinical data of patients, and to determine the factors that may be effective in the formation and spread of infection, antibiotic susceptibilities of Acinetobacter strains were evaluated by the Kirby Bauer disk diffusion method. Molecular typing was studied with PFGE.
\end{abstract}

Results: Netilmicin had the least resistance (7\%) among the strains, while the highest resistance was found against piperacillin (92\%), ceftriaxone (81\%), and doxycycline $(69 \%)$. When resistance profiles of strains were compared according to year (2005-2007), no significant changes were found for resistance rates to piperacillin, ceftriaxone, gentamicin, doxycycline, and trimethoprim / sulfamethoxazole. Of all the 131 tested Acinetobacter spp., $82(62.6 \%)$ of the strains were clustered with the PFGE method and $72.3 \%$ of the strains were clonally related. The increase in MDR, XDR and PDR rates among the isolated Acinetobacter spp. is remarkable. It was determined that the degree of transmission of the strains was quite high among patients. The clone in the hospital was able to remain in the environment for long periods and the general patient mortality rates were higher than other studies.

Conclusions: According to results and with the support of molecular typing studies, the need for more effective prevention and control measures in our hospital was demonstrated.

Key words: Acinetobacter, PFGE, cluster, resistant

Suggested Citation: Calıskan A, Durmaz R, Gursoy Ates C, Ildiz N. Investigation of Resistance and Clonal Relatedness Among Nosocomial Acinetobacter Isolates. Middle Black Sea Journal of Health Science, 2019; 5(2):120-132

\section{Address for correspondence/reprints:}

Nilay Ildız

Telephone number: +90 (352) 2076666

E-mail: nilaygucluer@erciyes.edu.tr

DOI: $10.19127 / \mathrm{mbsjohs.581706}$

\section{Introduction}

Acinetobacter species are important opportunistic pathogens that are responsible for severe hospital infections in various units, especially in intensive care units. It may cause various infections such as endotracheal tube- or tracheostomy-associated pneumonia, endocarditis, meningitis, skin and wound infections, peritonitis and urinary tract infections. Sporadic cases such as conjunctivitis, osteomyelitis and synovitis have also 
been reported (Buxton et al., 1978; Lyons, 1985; Bergogne-Berezin and Towner, 1996; Villegas and Harstein, 2003). Acinetobacter species play an important role in colonization and infection of hospitalized patients. Trauma, mechanical ventilation, and surgical procedures are important risk factors for nosocomial infections of this microorganism (Bergogne-Berezin and Towner, 1996; Parvez and Jarvis, 1999).

A. baumannii is the most common bacteria responsible for hospital-acquired infections (BeckSague et al., 1990; Lortholary et al., 1995; Villegas and Harstein, 2003). Other species such as $A$. johnsonii, A. lwoffii and A. radioresistens occur naturally on human skin and can also be found commonly in the oropharynx and vagina. Compared to other Acinetobacter species, A. lwoffii is more commonly associated with meningitis (SiegmanIgra et al., 1993).

The number of multidrug-resistant $A$. baumannii infections as opportunistic pathogens has increased globally in recent years. It has become one of the most difficult species to control and treat among nosocomial gram-negative pathogens (Jain and Danziger, 2004; Li et al., 2005). Patients infected with A. baumannii are often immunocompromised or have severe disease status and it is associated with mortality (Lyons, 1985; Bergogne-Berezin and Towner, 1996). Many of the outbreaks of $A$. baumannii have environmental origin, such as patient beds, air conditioners, and mechanical ventilation equipment (Villegas and Harstein, 2003). Acinetobacter spp. can survive for between 3 days to 5 months on dry inanimate surfaces (BeckSague et al., 1990; Siegman-Igra et al., 1993; Lortholary et al., 1995). This contributes to the emergence of outbreaks. In hospitalized patients and in various outbreaks, cases with high colonization in the skin, throat, respiratory system and digestive system were recorded. In patients receiving mechanical ventilation in intensive care units, colonization of the airways is very high due to contamination of these devices. Skin contamination is also common in these patients and is transmitted and spread to the hands of health personnel. Also, oropharyngeal and digestive tract colonization of Acinetobacter spp. is an important reservoir for outbreaks in patients hospitalized in intensive care units (Bergogne-Berezin and Towner, 1996; Corbella et al., 1996; Webster et al., 1998).

Acinetobacter species tend to resist many antibiotics. Almost all group members are resistant to penicillin, ampicillin and cephalothin and most of the strains are resistant to chloramphenicol (Seifert et al. 1993). Sensitivities to second and third generation cephalosporins and trimethoprim / sulfamethoxazole have been reported to be variable. In recent years, resistance to aminoglycosides has tended to increase among Acinetobacter species. Again, in nosocomial outbreaks, an increase is seen in strains showing multidrug resistance, including carbapenem-resistant Acinetobacter species (Bou et al., 2000; Hsueh et al., 2002). No international consensus seems to have been achieved yet for terms used to describe resistance in gram negative bacteria. The general trend in definition of antibiotic resistance is multi drug resistant (MDR) when resistant to $\geq 3$ drug groups (Giske et al., 2008). The term "extreme resistance, extensive resistance" (XDR) was adopted for resistance to all antibiotics except tigecycline and colistin. For resistance to all existing antibiotics, including tigecycline and colistin, "pan drug resistance" (PDR) is used (Falagas et al., 2005; Giske et al., 2008).

The increase of $A$. baumannii infections is due to incorrect infection control applications (Villegas and Harstein, 2003). In addition to the classical epidemiological information in the prevention of outbreaks, strain typing methods that provide determinative information about the source and transmission pathways of the causative microorganism are also important. Strain typing reveals the relationship between different sources in which microorganisms are isolated. Typing methods are important for defining the source and spread of epidemic strains. Pathogens associated with an epidemic have been identified by using molecular typing methods that support clinicalepidemiological data. A hypothesis is then generated based on the cause-and-effect relationship for the epidemiology of infection, including the shape of the contamination (reservoir and vector) and specific control measures. Continuous surveillance is essential to assess the effectiveness of the infection control measures and the treatment regimen (Jarvis, 1994; Aparajita et al., 2006).

The determination of the epidemiological relationship between nosocomial pathogens isolated from different sources in the past was based on the comparison of phenotypic characteristics such as biotype, serotype, bacteriophage or bacteriocin types and antimicrobial susceptibility profiles. This approach has begun to change over the last 20 years with new DNA-based technologies or developments in molecular analysis applications. For DNA-based molecular typing, Pulsed Field Gel Electrophoresis 
(PFGE) and other restriction-based methods consist of plasmid analysis and polymerase chain reaction (PCR) based typing methods (Aber and Mackel, 1981; Goering, 1993; Arbeit, 1995).

In this study, Acinetobacter spp. were isolated from inpatients in various wards in a research hospital. The aim of this study was to determine the resistance rate of the strains to antibiotics, to observe the increase in resistance during the years, to determine whether there is clonal relationship between the strains by PFGE method, to evaluate the epidemiological and clinical data of the patients, and to determine the factors that may be effective in the formation and spread of infection.

\section{Methods}

Acinetobacter spp. and Patient selection criteria. A total of 135 Acinetobacter strains belonging to 130 patients were recruited from January 2005 to December 2007 in İnönü University Turgut Özal Medical Center. All patients were evaluated by the Infection Control Committee in our hospital and it was decided whether they had a hospital infection according to the criteria of the Centers for Disease Control and Prevention (CDC) in the United States (CLSI, 2008).

\section{Evaluation of Epidemiological Relationship} and Risk Factors. For each patient with accepted hospital infection, an epidemiological study form was prepared with some questions such as gender, age, hospital ward, clinical diagnosis, sample type, and culture results.

Identification of strains. Clinical specimens taken from hospitalized patients were inoculated in blood agar and kept for 24 hours at $35^{\circ} \mathrm{C}$. Gram staining, colony morphologies, oxidase and sugar fermentation activities, and catalase reactions of Acinetobacter spp. were investigated. Identification of strains was performed by using a Phoenix 100 system (Becton Dickinson Microbiology Systems, USA).

Antibiotic Susceptibility Profiles of Strains. The antibiotic susceptibilities of Acinetobacter strains isolated from clinical samples were investigated according to Clinical \& Laboratory Standards Institute: CLSI Guidelines using the Kirby Bauer disk diffusion method (CLSI, 2008).

Imipenem resistant strains were confirmed with the imipenem E-test method. Acinetobacter spp. strains with the probability of pandrug resistance were evaluated by the disc diffusion method for sensitivity to colistin and tigecycline. The colistin sensitivity was evaluated according to the zone diameter in nonfermentary bacteria and for tigecycline sensitivity the study by Jones and colleagues (2007) was used as a reference.

Molecular Typing. Acinetobacter strains which were grown from the samples of patients who were hospitalized in the wards were examined by PFGE. The protocol of Durmaz et al. (2007) was used for the PFGE application.

Monitoring and Analysis of Results. After electrophoresis, the gel was taken up in $400 \mathrm{ml}$ of ultrapure water containing $5 \mathrm{mg} / \mathrm{ml}$ ethidium bromide and stained for 20 minutes. Gel images were observed under UV light. Images of DNA band strips were taken using Gel Logic 2200 Imaging System (1708×1280 pixel, Kodak Company, NY, USA). Images were saved in TIFF format. Band profiles were analyzed using the GelCompar II software system (version 3.0; Applied Maths, Sint-Martens-Latem, Belgium). First of all, normalization between the images was done with the help of three standards $(1,7$, and 15th wells) in each image. The dendrogram of the PFGE profiles were generated using "Unweighted pair group method with mathematical averaging (UPGMA)" and clustering analysis was completed. The relationship between the strains was determined according to the Dice similarity coefficient. In the calculation of the similarity coefficient, band and profile tolerance was taken as $1-1.5 \%$. Using criteria developed by Tenover et al. (1997), isolates were considered to be identical, closely related, possibly related, and unrelated.

Statistical analyses. Pearson's chi-square analysis (exact test) was used to determine the relationships between categorical variables. The analysis of the data was performed with the statistical software TURCOSA (Turcosa Analytical Solutions Ltd., www.turcosa.com.tr). " $p<0.05$ ” was accepted as statistically significant.

\section{Results}

Epidemiological Results. Of Acinetobacter strains evaluated as infectious agents, 131 (97\%) were identified as A. baumannii and $4(3 \%)$ as $A$. lwoffi. Of all the strains, $1 / 3$ of $A$. baumannii isolates were isolated from tracheal aspiration culture and about 1/4 were isolated from blood culture. Two Acinetobacter baumannii were isolated from 
cerebrospinal fluid (CSF) culture of patients hospitalized in the neurosurgery and neurosurgery intensive care unit. Two patients with A. baumannii isolated underwent invasive procedures. One patient had immunodeficiency and the other patient had Arnold-Chiari syndrome. Unlike other studies, A. lwoffii was not isolated as a meningitis agent.

Antibiotic Susceptibility Results. In this study, the most effective antibiotics against the strains that tested were netilmicin $(7 \%)$ and the least effective antibiotics were piperacillin (92\%), ceftriaxone (81\%), doxycycline (69\%), and gentamicin (67\%).

According to year, comparison of the change in antibiotic resistance profiles for Acinetobacter spp. found no significant change in resistance rates to piperacillin, ceftriaxone, gentamicin, doxycycline, and trimethoprim / sulfamethoxazole. When we compared 2005 and 2007, the resistance rate rose from $47 \%$ to $76 \%$ for piperacillin / tazobactam, rose from $42 \%$ to $77 \%$ for cefepime, rose from $47 \%$ to $77 \%$ for ceftazidime, rose from zero to $39 \%$ for imipenem, rose from zero to $14 \%$ for netilmicin, rose from $18 \%$ to $53 \%$ for amikacin, rose from $18 \%$ to $47 \%$ for tobramycin, rose from $24 \%$ to $55 \%$ for levofloxacin, and rose from $16 \%$ to $46 \%$ for ampicillin/sulbactam. The antibiotic resistance profiles of A. baumannii isolates changed over time.

Table 1. Statistical analyses of antibiotics relation with time

Table 2. Antibiotic susceptibility profiles of isolates in the same cluster
Molecular Typing Results. As a result of PFGE typing, about three quarters of the strains were found to be clonally related. Cluster-forming strains were predominantly isolated from respiratory $(42.7 \%)$ and blood culture $(29.3 \%)$ samples. The number of strains increased to 27 in some clusters. This situation demonstrates the magnitude of the cross-contamination severity. Twenty-seven strains in this cluster were present in our hospital for approximately 9 months. The duration of other clusters in hospital extended to 27 months. These data emphasize that an important nosocomial pathogen, such as A. baumannii, can be easily disseminated in the hospital and can survive in the hospital environment for many years if proper prevention and control are not ensured.

Clonally associated strains displayed variety in susceptibility profiles to drugs over time. Our twelfth cluster was also found to be the most antibiotic-resistant cluster. All isolates found in this cluster were found to be MDR. Only two of the isolates in the cluster were susceptible to imipenem (Table 1). Significant changes in the susceptibility profiles of the strains found in the cluster were recorded (Table 1).

\begin{tabular}{|c|c|c|c|c|c|}
\hline & 2005 & 2006 & 2007 & $p$ & $2005-2007$ \\
\hline Antibiotics & $N=38$ & $\mathrm{~N}=23$ & $N=74$ & & $N=135$ \\
\hline PIP & $34(89.5)$ & $19(82.6)$ & $70(94.6)$ & 0.168 & 92 \\
\hline TZP & $20(52.6)$ & $12(52.2)$ & $56(75.7)$ & 0.019 & 64 \\
\hline FEP & $16(57.1)$ & $14(60.9)$ & $57(77.0)$ & 0.087 & 64 \\
\hline CAZ & $18(47.4)$ & $14(60.9)$ & $57(77.0)$ & 0.006 & 66 \\
\hline CRO & $30(78.9)$ & $17(73.9)$ & $62(83.8)$ & 0.551 & 81 \\
\hline İPM & $38(100.0)$ & $5(21.7)$ & $29(39.2)$ & $<0.001$ & 25 \\
\hline NET & $38(100.0)$ & $23(100.0)$ & 13(17.6) & $<0.001$ & 7 \\
\hline GM & $25(65.8)$ & $11(47.8)$ & $55(74.3)$ & 0.050 & 67 \\
\hline AK & $7(18.4)$ & $5(30.4)$ & $39(52.7)$ & 0.001 & 39 \\
\hline TOB & $7(18.4)$ & $5(21.7)$ & $35(47.3)$ & 0.004 & 35 \\
\hline DOX & $26(68.4)$ & $19(82.6)$ & $49(66.2)$ & 0.340 & 69 \\
\hline LVX & $9(23.7)$ & $11(47.8)$ & 41(48.8) & 0.028 & 45 \\
\hline SXT & $25(65.8)$ & $14(60.9)$ & $51(60.7)$ & 0.888 & 67 \\
\hline SAM & $7(18.4)$ & 1net1(47.8) & $34(45.9)$ & 0.011 & 38 \\
\hline
\end{tabular}




\begin{tabular}{|c|c|c|c|c|c|c|c|c|c|c|c|c|c|c|c|}
\hline Stock no & $\begin{array}{c}\text { PFGE } \\
\text { type }\end{array}$ & PIP & TZP & FEP & CAZ & CRO & IMP & NET & GM & $\mathbf{A K}$ & TOB & DOX & LVX & SXT & SAM \\
\hline 28 & V & $\mathbf{R}$ & $\mathbf{R}$ & $\mathbf{R}$ & $\mathbf{R}$ & $\mathbf{R}$ & $\mathbf{S}$ & $\mathbf{S}$ & $\mathbf{R}$ & I & $\mathbf{R}$ & $\mathbf{R}$ & $\mathbf{R}$ & $\mathbf{R}$ & $\mathbf{S}$ \\
\hline 35 & $\mathbf{V}$ & $\mathbf{R}$ & $\mathbf{R}$ & I & $\mathbf{R}$ & $\mathbf{R}$ & $\mathbf{S}$ & $\mathbf{S}$ & $\mathbf{R}$ & I & $\mathbf{R}$ & $\mathbf{R}$ & $\mathbf{R}$ & $\mathbf{R}$ & $\mathbf{S}$ \\
\hline 22 & V-a & $\mathbf{R}$ & $\mathbf{R}$ & $\mathbf{I}$ & $\mathbf{R}$ & $\mathbf{R}$ & $\mathbf{S}$ & $\mathbf{S}$ & $\mathbf{R}$ & I & $\mathbf{R}$ & $\mathbf{R}$ & $\mathbf{R}$ & $\mathbf{R}$ & $\mathbf{S}$ \\
\hline 17 & V-b & $\mathbf{R}$ & $\mathbf{R}$ & $\mathbf{R}$ & $\mathbf{R}$ & $\mathbf{R}$ & $\mathbf{R}$ & $\mathbf{S}$ & $\mathbf{R}$ & $\mathbf{R}$ & $\mathbf{R}$ & $\mathbf{S}$ & $\mathbf{R}$ & $\mathbf{R}$ & $\mathbf{R}$ \\
\hline 24 & VII & $\mathbf{R}$ & $\mathbf{R}$ & $\mathbf{R}$ & $\mathbf{R}$ & $\mathbf{R}$ & $\mathbf{R}$ & $\mathbf{S}$ & $\mathbf{R}$ & $\mathbf{R}$ & $\mathbf{S}$ & $\mathbf{R}$ & $\mathbf{R}$ & $\mathbf{R}$ & $\mathbf{R}$ \\
\hline 27 & VII & $\mathbf{R}$ & $\mathbf{R}$ & $\mathbf{R}$ & $\mathbf{R}$ & $\mathbf{R}$ & $\mathbf{S}$ & $\mathbf{S}$ & $\mathbf{R}$ & $\mathbf{S}$ & $\mathbf{S}$ & $\mathbf{R}$ & $\mathbf{R}$ & $\mathbf{R}$ & $\mathbf{R}$ \\
\hline 85 & VII-a & $\mathbf{R}$ & $\mathbf{R}$ & $\mathbf{R}$ & $\mathbf{R}$ & $\mathbf{R}$ & $\mathbf{S}$ & $\mathbf{S}$ & $\mathbf{R}$ & $\mathbf{S}$ & $\mathbf{S}$ & I & $\mathbf{R}$ & I & $\mathbf{R}$ \\
\hline 86 & VII-a & $\mathbf{R}$ & $\mathbf{R}$ & $\mathbf{R}$ & $\mathbf{R}$ & $\mathbf{R}$ & $\mathbf{S}$ & $\mathbf{S}$ & $\mathbf{R}$ & $\mathbf{S}$ & $\mathbf{S}$ & $\mathbf{S}$ & $\mathbf{S}$ & $\mathbf{R}$ & $\mathbf{R}$ \\
\hline 34 & VII-b & $\mathbf{R}$ & $\mathbf{R}$ & $\mathbf{R}$ & $\mathbf{R}$ & $\mathbf{R}$ & $\mathbf{S}$ & I & $\mathbf{R}$ & $\mathbf{S}$ & I & $\mathbf{R}$ & $\mathbf{R}$ & $\mathbf{R}$ & $\mathbf{R}$ \\
\hline 93 & VII-b & $\mathbf{R}$ & $\mathbf{R}$ & $\mathbf{R}$ & $\mathbf{R}$ & $\mathbf{R}$ & $\mathbf{S}$ & $\mathbf{S}$ & $\mathbf{R}$ & $\mathbf{S}$ & $\mathbf{S}$ & $\mathbf{S}$ & $\mathbf{S}$ & $\mathbf{S}$ & $\mathbf{R}$ \\
\hline 21 & VIII & $\mathbf{R}$ & I & I & I & $\mathbf{R}$ & $\mathbf{S}$ & $\mathbf{S}$ & $\mathbf{R}$ & $\mathbf{S}$ & $\mathbf{S}$ & $\mathbf{R}$ & $\mathbf{S}$ & $\mathbf{R}$ & I \\
\hline 52 & VIII & $\mathbf{R}$ & $\mathbf{R}$ & $\mathbf{R}$ & $\mathbf{R}$ & $\mathbf{R}$ & $\mathbf{S}$ & $\mathbf{S}$ & $\mathbf{S}$ & $\mathbf{S}$ & $\mathbf{S}$ & $\mathbf{R}$ & $\mathbf{S}$ & $\mathbf{S}$ & $\mathbf{S}$ \\
\hline 16 & VIII-a & $\mathbf{R}$ & $\mathbf{S}$ & $\mathbf{S}$ & I & $\mathbf{R}$ & $\mathbf{S}$ & $\mathbf{S}$ & $\mathbf{R}$ & $\mathbf{S}$ & $\mathbf{S}$ & $\mathbf{R}$ & $\mathbf{S}$ & $\mathbf{S}$ & $\mathbf{S}$ \\
\hline 2 & IX & $\mathbf{R}$ & $\mathbf{R}$ & $\mathbf{R}$ & $\mathbf{R}$ & $\mathbf{R}$ & $\mathbf{S}$ & $\mathbf{S}$ & $\mathbf{R}$ & $\mathbf{S}$ & $\mathbf{S}$ & I & $\mathbf{R}$ & $\mathbf{S}$ & $\mathbf{R}$ \\
\hline 11 & IX & $\mathbf{R}$ & $\mathbf{R}$ & $\mathbf{R}$ & $\mathbf{R}$ & $\mathbf{R}$ & $\mathbf{S}$ & $\mathbf{S}$ & $\mathbf{R}$ & $\mathbf{S}$ & $\mathbf{S}$ & $\mathbf{R}$ & $\mathbf{S}$ & $\mathbf{S}$ & $\mathbf{R}$ \\
\hline 10 & IX & $\mathbf{R}$ & $\mathbf{R}$ & $\mathbf{R}$ & $\mathbf{R}$ & $\mathbf{R}$ & $\mathbf{S}$ & $\mathbf{S}$ & $\mathbf{S}$ & $\mathbf{S}$ & $\mathbf{S}$ & $\mathbf{R}$ & $\mathbf{R}$ & $\mathbf{S}$ & $\mathbf{S}$ \\
\hline 19 & IX & $\mathbf{R}$ & I & $\mathbf{R}$ & $\mathbf{R}$ & $\mathbf{R}$ & $\mathbf{S}$ & $\mathbf{S}$ & $\mathbf{S}$ & $\mathbf{S}$ & $\mathbf{S}$ & $\mathbf{R}$ & $\mathbf{R}$ & I & $\mathbf{S}$ \\
\hline 20 & IX & $\mathbf{R}$ & $\mathbf{R}$ & $\mathbf{R}$ & $\mathbf{R}$ & $\mathbf{R}$ & $\mathbf{S}$ & $\mathbf{S}$ & $\mathbf{S}$ & $\mathbf{S}$ & $\mathbf{S}$ & $\mathbf{R}$ & $\mathbf{R}$ & $\mathbf{S}$ & $\mathbf{S}$ \\
\hline 65 & IX & $\mathbf{R}$ & $\mathbf{R}$ & $\mathbf{R}$ & $\mathbf{R}$ & $\mathbf{R}$ & $\mathbf{S}$ & $\mathbf{S}$ & $\mathbf{R}$ & $\mathbf{S}$ & $\mathbf{S}$ & $\mathbf{S}$ & $\mathbf{S}$ & $\mathbf{S}$ & $\mathbf{R}$ \\
\hline 66 & IX & $\mathbf{R}$ & $\mathbf{R}$ & $\mathbf{R}$ & $\mathbf{R}$ & $\mathbf{R}$ & $\mathbf{S}$ & $\mathbf{S}$ & $\mathbf{R}$ & $\mathbf{S}$ & $\mathbf{S}$ & $\mathbf{S}$ & $\mathbf{S}$ & $\mathbf{S}$ & $\mathbf{R}$ \\
\hline 67 & IX & $\mathbf{R}$ & $\mathbf{R}$ & $\mathbf{R}$ & $\mathbf{R}$ & $\mathbf{R}$ & $\mathbf{S}$ & $\mathbf{S}$ & $\mathbf{R}$ & $\mathbf{S}$ & $\mathbf{S}$ & $\mathbf{S}$ & I & $\mathbf{S}$ & $\mathbf{R}$ \\
\hline 80 & IX & $\mathbf{R}$ & $\mathbf{S}$ & $\mathbf{R}$ & $\mathbf{R}$ & $\mathbf{R}$ & $\mathbf{S}$ & $\mathbf{S}$ & $\mathbf{R}$ & $\mathbf{S}$ & I & $\mathbf{S}$ & $\mathbf{S}$ & $\mathbf{S}$ & $\mathbf{R}$ \\
\hline 103 & IX & $\mathbf{R}$ & $\mathbf{R}$ & $\mathbf{R}$ & $\mathbf{R}$ & $\mathbf{R}$ & $\mathbf{S}$ & $\mathbf{R}$ & $\mathbf{S}$ & $\mathbf{S}$ & $\mathbf{S}$ & I & $\mathbf{R}$ & $\mathbf{S}$ & I \\
\hline 138 & IX & $\mathbf{R}$ & $\mathbf{R}$ & $\mathbf{R}$ & $\mathbf{R}$ & $\mathbf{R}$ & $\mathbf{S}$ & $\mathbf{S}$ & $\mathbf{R}$ & $\mathbf{R}$ & $\mathbf{S}$ & $\mathbf{R}$ & $\mathbf{S}$ & $\mathbf{R}$ & $\mathbf{R}$ \\
\hline 30 & $\mathbf{X}$ & $\mathbf{R}$ & $\mathbf{S}$ & $\mathbf{S}$ & $\mathbf{S}$ & I & $\mathbf{S}$ & $\mathbf{S}$ & $\mathbf{S}$ & $\mathbf{S}$ & $\mathbf{S}$ & $\mathbf{R}$ & $\mathbf{S}$ & $\mathbf{R}$ & $\mathbf{S}$ \\
\hline 60 & $\mathbf{X}$ & $\mathbf{R}$ & $\mathbf{S}$ & $\mathbf{S}$ & $\mathbf{S}$ & I & $\mathbf{S}$ & $\mathbf{S}$ & $\mathbf{S}$ & $\mathbf{S}$ & $\mathbf{S}$ & $\mathbf{S}$ & $\mathbf{S}$ & $\mathbf{S}$ & $\mathbf{S}$ \\
\hline 5 & XII & $\mathbf{R}$ & $\mathbf{R}$ & $\mathbf{R}$ & $\mathbf{R}$ & $\mathbf{R}$ & $\mathbf{S}$ & $\mathbf{S}$ & $\mathbf{R}$ & $\mathbf{S}$ & $\mathbf{S}$ & $\mathbf{R}$ & $\mathbf{S}$ & $\mathbf{R}$ & I \\
\hline 7 & XII & $\mathbf{R}$ & I & I & I & $\mathbf{R}$ & $\mathbf{S}$ & $\mathbf{S}$ & $\mathbf{S}$ & $\mathbf{S}$ & $\mathbf{S}$ & $\mathbf{R}$ & $\mathbf{S}$ & $\mathbf{R}$ & I \\
\hline 12 & XII & $\mathbf{R}$ & I & I & I & $\mathbf{R}$ & $\mathbf{S}$ & $\mathbf{S}$ & $\mathbf{R}$ & $\mathbf{R}$ & $\mathbf{S}$ & $\mathbf{R}$ & $\mathbf{S}$ & $\mathbf{R}$ & I \\
\hline 13 & XII & $\mathbf{R}$ & $\mathbf{R}$ & $\mathbf{R}$ & $\mathbf{R}$ & $\mathbf{R}$ & $\mathbf{S}$ & $\mathbf{S}$ & $\mathbf{R}$ & $\mathbf{R}$ & $\mathbf{S}$ & $\mathbf{R}$ & $\mathbf{S}$ & $\mathbf{R}$ & I \\
\hline 14 & XII & $\mathbf{R}$ & $\mathbf{R}$ & $\mathbf{R}$ & $\mathbf{R}$ & $\mathbf{R}$ & $\mathbf{S}$ & $\mathbf{S}$ & $\mathbf{R}$ & $\mathbf{R}$ & $\mathbf{S}$ & $\mathbf{R}$ & $\mathbf{S}$ & $\mathbf{R}$ & I \\
\hline 15 & XII & $\mathbf{R}$ & $\mathbf{R}$ & $\mathbf{R}$ & $\mathbf{R}$ & $\mathbf{R}$ & $\mathbf{S}$ & $\mathbf{S}$ & $\mathbf{R}$ & $\mathbf{S}$ & $\mathbf{S}$ & $\mathbf{S}$ & $\mathbf{S}$ & $\mathbf{R}$ & $\mathbf{S}$ \\
\hline 25 & XII & $\mathbf{R}$ & $\mathbf{R}$ & I & I & $\mathbf{R}$ & $\mathbf{S}$ & $\mathbf{S}$ & $\mathbf{R}$ & $\mathbf{S}$ & $\mathbf{S}$ & $\mathbf{R}$ & $\mathbf{S}$ & $\mathbf{R}$ & I \\
\hline 40 & XII & $\mathbf{R}$ & I & I & I & $\mathbf{R}$ & $\mathbf{S}$ & $\mathbf{S}$ & $\mathbf{R}$ & $\mathbf{S}$ & $\mathbf{S}$ & $\mathbf{R}$ & $\mathbf{S}$ & $\mathbf{R}$ & I \\
\hline 53 & XII & $\mathbf{R}$ & $\mathbf{R}$ & $\mathbf{R}$ & $\mathbf{R}$ & $\mathbf{R}$ & $\mathbf{S}$ & $\mathbf{S}$ & $\mathbf{R}$ & $\mathbf{R}$ & $\mathbf{S}$ & $\mathbf{S}$ & $\mathbf{S}$ & $\mathbf{R}$ & $\mathbf{S}$ \\
\hline 63 & XII & $\mathbf{R}$ & I & $\mathbf{R}$ & I & $\mathbf{R}$ & $\mathbf{S}$ & $\mathbf{S}$ & $\mathbf{R}$ & $\mathbf{S}$ & $\mathbf{S}$ & $\mathbf{R}$ & $\mathbf{S}$ & $\mathbf{R}$ & I \\
\hline 125 & XII & $\mathbf{R}$ & I & I & I & $\mathbf{R}$ & $\mathbf{S}$ & $\mathbf{S}$ & I & $\mathbf{S}$ & $\mathbf{S}$ & $\mathbf{S}$ & $\mathbf{R}$ & $\mathbf{S}$ & $\mathbf{S}$ \\
\hline 127 & XII & $\mathbf{R}$ & $\mathbf{R}$ & $\mathbf{R}$ & $\mathbf{R}$ & $\mathbf{R}$ & $\mathbf{R}$ & $\mathbf{S}$ & $\mathbf{R}$ & $\mathbf{R}$ & $\mathbf{R}$ & $\mathbf{R}$ & $\mathbf{R}$ & $\mathbf{R}$ & I \\
\hline 1 & XII-a & $\mathbf{R}$ & $\mathbf{R}$ & $\mathbf{R}$ & $\mathbf{R}$ & $\mathbf{R}$ & $\mathbf{S}$ & $\mathbf{S}$ & $\mathbf{S}$ & $\mathbf{S}$ & $\mathbf{S}$ & $\mathbf{R}$ & $\mathbf{S}$ & I & $\mathbf{S}$ \\
\hline 33 & XII-a & $\mathbf{R}$ & $\mathbf{R}$ & $\mathbf{R}$ & $\mathbf{R}$ & $\mathbf{R}$ & $\mathbf{S}$ & $\mathbf{S}$ & $\mathbf{S}$ & $\mathbf{S}$ & $\mathbf{S}$ & $\mathbf{R}$ & $\mathbf{S}$ & $\mathbf{S}$ & $\mathbf{S}$ \\
\hline 58 & XII-a & $\mathbf{R}$ & $\mathbf{R}$ & I & I & $\mathbf{R}$ & $\mathbf{S}$ & $\mathbf{S}$ & $\mathbf{R}$ & $\mathbf{S}$ & $\mathbf{R}$ & $\mathbf{R}$ & $\mathbf{S}$ & $\mathbf{R}$ & I \\
\hline 114 & XII-a1 & $\mathbf{R}$ & $\mathbf{R}$ & I & I & $\mathbf{R}$ & $\mathbf{S}$ & $\mathbf{S}$ & $\mathbf{R}$ & I & $\mathbf{S}$ & $\mathbf{S}$ & $\mathbf{S}$ & $\mathbf{R}$ & I \\
\hline 50 & XIII & $\mathbf{R}$ & I & $\mathbf{R}$ & $\mathbf{R}$ & $\mathbf{R}$ & $\mathbf{S}$ & $\mathbf{S}$ & $\mathbf{R}$ & $\mathbf{S}$ & $\mathbf{S}$ & $\mathbf{S}$ & $\mathbf{S}$ & $\mathbf{S}$ & $\mathbf{S}$ \\
\hline 38 & XIII-b & $\mathbf{R}$ & $\mathbf{S}$ & I & I & $\mathbf{R}$ & $\mathbf{S}$ & $\mathbf{S}$ & $\mathbf{S}$ & $\mathbf{S}$ & $\mathbf{S}$ & $\mathbf{R}$ & $\mathbf{R}$ & $\mathbf{R}$ & $\mathbf{S}$ \\
\hline 137 & $\mathbf{X X}$ & $\mathbf{R}$ & $\mathbf{R}$ & $\mathbf{R}$ & $\mathbf{R}$ & $\mathbf{R}$ & $\mathbf{S}$ & $\mathbf{R}$ & $\mathbf{R}$ & $\mathbf{R}$ & $\mathbf{R}$ & $\mathbf{R}$ & $\mathbf{R}$ & $\mathbf{R}$ & $\mathbf{S}$ \\
\hline 140 & $\mathbf{X X}$ & $\mathbf{R}$ & $\mathbf{R}$ & $\mathbf{R}$ & $\mathbf{R}$ & $\mathbf{R}$ & $\mathbf{S}$ & $\mathbf{R}$ & $\mathbf{S}$ & I & $\mathbf{R}$ & $\mathbf{R}$ & $\mathbf{R}$ & $\mathbf{R}$ & I \\
\hline 68 & XXIX & $\mathbf{R}$ & $\mathbf{R}$ & $\mathbf{R}$ & $\mathbf{R}$ & $\mathbf{R}$ & $\mathbf{R}$ & I & $\mathbf{R}$ & $\mathbf{R}$ & $\mathbf{R}$ & $\mathbf{R}$ & $\mathbf{R}$ & $\mathbf{R}$ & I \\
\hline 70 & XXIX & $\mathbf{R}$ & $\mathbf{R}$ & $\mathbf{R}$ & $\mathbf{R}$ & $\mathbf{R}$ & $\mathbf{R}$ & $\mathbf{S}$ & $\mathbf{R}$ & $\mathbf{R}$ & $\mathbf{R}$ & $\mathbf{R}$ & $\mathbf{R}$ & $\mathbf{R}$ & $\mathbf{R}$ \\
\hline 72 & XXIX & $\mathbf{R}$ & $\mathbf{R}$ & $\mathbf{R}$ & $\mathbf{R}$ & $\mathbf{R}$ & $\mathbf{R}$ & I & $\mathbf{R}$ & $\mathbf{S}$ & $\mathbf{R}$ & $\mathbf{R}$ & $\mathbf{R}$ & $\mathbf{R}$ & $\mathbf{S}$ \\
\hline 73 & XXIX & $\mathbf{R}$ & $\mathbf{R}$ & $\mathbf{R}$ & $\mathbf{R}$ & $\mathbf{R}$ & $\mathbf{S}$ & $\mathbf{S}$ & $\mathbf{R}$ & $\mathbf{R}$ & $\mathbf{R}$ & $\mathbf{R}$ & $\mathbf{R}$ & $\mathbf{R}$ & I \\
\hline 75 & XXIX & $\mathbf{R}$ & $\mathbf{R}$ & $\mathbf{R}$ & $\mathbf{R}$ & $\mathbf{R}$ & $\mathbf{R}$ & $\mathbf{S}$ & $\mathbf{R}$ & $\mathbf{R}$ & $\mathbf{R}$ & $\mathbf{R}$ & $\mathbf{R}$ & $\mathbf{R}$ & I \\
\hline 76 & XXIX & $\mathbf{R}$ & $\mathbf{R}$ & $\mathbf{R}$ & $\mathbf{R}$ & $\mathbf{R}$ & $\mathbf{R}$ & $\mathbf{S}$ & $\mathbf{R}$ & $\mathbf{R}$ & $\mathbf{R}$ & $\mathbf{R}$ & $\mathbf{R}$ & $\mathbf{R}$ & $\mathbf{R}$ \\
\hline
\end{tabular}




\begin{tabular}{|c|c|c|c|c|c|c|c|c|c|c|c|c|c|c|c|}
\hline 79 & XXIX & $\mathbf{R}$ & $\mathbf{R}$ & $\mathbf{R}$ & $\mathbf{R}$ & $\mathbf{R}$ & $\mathbf{R}$ & $\mathbf{S}$ & $\mathbf{R}$ & $\mathbf{R}$ & $\mathbf{R}$ & $\mathbf{R}$ & $\mathbf{R}$ & $\mathbf{R}$ & $\mathbf{R}$ \\
\hline 83 & XXIX & $\mathbf{R}$ & $\mathbf{R}$ & $\mathbf{R}$ & $\mathbf{R}$ & $\mathbf{R}$ & $\mathbf{R}$ & $\mathbf{R}$ & $\mathbf{R}$ & $\mathbf{R}$ & $\mathbf{R}$ & $\mathbf{R}$ & $\mathbf{R}$ & $\mathbf{R}$ & $\mathbf{R}$ \\
\hline 91 & XXIX & $\mathbf{R}$ & $\mathbf{R}$ & $\mathbf{R}$ & $\mathbf{R}$ & $\mathbf{R}$ & $\mathbf{R}$ & $\mathbf{S}$ & $\mathbf{R}$ & $\mathbf{R}$ & $\mathbf{R}$ & $\mathbf{R}$ & $\mathbf{R}$ & $\mathbf{R}$ & $\mathbf{R}$ \\
\hline 92 & XXIX & $\mathbf{R}$ & $\mathbf{R}$ & $\mathbf{R}$ & $\mathbf{R}$ & $\mathbf{R}$ & $\mathbf{R}$ & $\mathbf{S}$ & $\mathbf{R}$ & $\mathbf{R}$ & $\mathbf{R}$ & $\mathbf{R}$ & $\mathbf{R}$ & $\mathbf{R}$ & $\mathbf{R}$ \\
\hline 97 & XXIX & $\mathbf{R}$ & $\mathbf{R}$ & $\mathbf{R}$ & $\mathbf{R}$ & $\mathbf{R}$ & $\mathbf{R}$ & $\mathbf{R}$ & $\mathbf{R}$ & $\mathbf{R}$ & $\mathbf{R}$ & $\mathbf{R}$ & $\mathbf{R}$ & $\mathbf{R}$ & $\mathbf{S}$ \\
\hline 98 & XXIX & $\mathbf{R}$ & $\mathbf{R}$ & $\mathbf{R}$ & $\mathbf{R}$ & $\mathbf{R}$ & $\mathbf{R}$ & I & $\mathbf{R}$ & $\mathbf{R}$ & $\mathbf{R}$ & $\mathbf{R}$ & $\mathbf{R}$ & $\mathbf{R}$ & I \\
\hline 102 & XXIX & $\mathbf{R}$ & $\mathbf{R}$ & $\mathbf{R}$ & $\mathbf{R}$ & $\mathbf{R}$ & $\mathbf{R}$ & $\mathbf{R}$ & $\mathbf{R}$ & $\mathbf{R}$ & $\mathbf{R}$ & $\mathbf{R}$ & $\mathbf{R}$ & $\mathbf{R}$ & $\mathbf{R}$ \\
\hline 106 & XXIX & $\mathbf{R}$ & $\mathbf{R}$ & $\mathbf{R}$ & $\mathbf{R}$ & $\mathbf{R}$ & $\mathbf{R}$ & $\mathbf{S}$ & $\mathbf{R}$ & $\mathbf{R}$ & $\mathbf{R}$ & $\mathbf{R}$ & $\mathbf{R}$ & $\mathbf{R}$ & $\mathbf{R}$ \\
\hline 109 & XXIX & $\mathbf{R}$ & $\mathbf{R}$ & $\mathbf{R}$ & $\mathbf{R}$ & $\mathbf{R}$ & $\mathbf{R}$ & $\mathbf{S}$ & $\mathbf{R}$ & $\mathbf{R}$ & $\mathbf{R}$ & $\mathbf{R}$ & $\mathbf{R}$ & $\mathbf{R}$ & $\mathbf{R}$ \\
\hline 111 & XXIX & $\mathbf{R}$ & $\mathbf{R}$ & $\mathbf{R}$ & $\mathbf{R}$ & $\mathbf{R}$ & $\mathbf{R}$ & $\mathbf{S}$ & $\mathbf{R}$ & $\mathbf{R}$ & $\mathbf{R}$ & $\mathbf{R}$ & $\mathbf{R}$ & $\mathbf{R}$ & $\mathbf{R}$ \\
\hline 113 & XXIX & $\mathbf{R}$ & $\mathbf{R}$ & $\mathbf{R}$ & $\mathbf{R}$ & $\mathbf{R}$ & $\mathbf{R}$ & I & $\mathbf{R}$ & $\mathbf{R}$ & $\mathbf{R}$ & $\mathbf{R}$ & $\mathbf{R}$ & $\mathbf{R}$ & $\mathbf{R}$ \\
\hline 115 & XXIX & $\mathbf{R}$ & $\mathbf{R}$ & $\mathbf{R}$ & $\mathbf{R}$ & $\mathbf{R}$ & $\mathbf{R}$ & I & $\mathbf{R}$ & $\mathbf{R}$ & $\mathbf{R}$ & $\mathbf{R}$ & $\mathbf{R}$ & $\mathbf{R}$ & $\mathbf{R}$ \\
\hline 118 & XXIX & $\mathbf{R}$ & $\mathbf{R}$ & $\mathbf{R}$ & $\mathbf{R}$ & $\mathbf{R}$ & $\mathbf{R}$ & $\mathbf{S}$ & $\mathbf{R}$ & $\mathbf{R}$ & $\mathbf{R}$ & $\mathbf{R}$ & $\mathbf{R}$ & $\mathbf{R}$ & $\mathbf{R}$ \\
\hline 119 & XXIX & $\mathbf{R}$ & $\mathbf{R}$ & $\mathbf{R}$ & $\mathbf{R}$ & $\mathbf{R}$ & $\mathbf{R}$ & I & $\mathbf{R}$ & $\mathbf{R}$ & $\mathbf{R}$ & $\mathbf{R}$ & $\mathbf{R}$ & $\mathbf{R}$ & $\mathbf{R}$ \\
\hline 120 & XXIX & $\mathbf{R}$ & $\mathbf{R}$ & $\mathbf{R}$ & $\mathbf{R}$ & $\mathbf{R}$ & $\mathbf{R}$ & $\mathbf{S}$ & $\mathbf{R}$ & $\mathbf{R}$ & $\mathbf{R}$ & $\mathbf{R}$ & $\mathbf{R}$ & $\mathbf{R}$ & $\mathbf{R}$ \\
\hline 123 & XXIX & $\mathbf{R}$ & $\mathbf{R}$ & $\mathbf{R}$ & $\mathbf{R}$ & $\mathbf{R}$ & $\mathbf{R}$ & $\mathbf{S}$ & $\mathbf{R}$ & $\mathbf{R}$ & $\mathbf{R}$ & $\mathbf{R}$ & $\mathbf{R}$ & $\mathbf{R}$ & $\mathbf{R}$ \\
\hline 132 & XXIX & $\mathbf{R}$ & $\mathbf{R}$ & $\mathbf{R}$ & $\mathbf{R}$ & $\mathbf{R}$ & $\mathbf{R}$ & $\mathbf{S}$ & $\mathbf{R}$ & $\mathbf{R}$ & $\mathbf{R}$ & $\mathbf{R}$ & $\mathbf{R}$ & $\mathbf{R}$ & I \\
\hline 142 & XXIX & $\mathbf{R}$ & $\mathbf{R}$ & $\mathbf{R}$ & $\mathbf{R}$ & $\mathbf{R}$ & $\mathbf{R}$ & $\mathbf{S}$ & $\mathbf{R}$ & $\mathbf{R}$ & $\mathbf{R}$ & $\mathbf{R}$ & $\mathbf{R}$ & $\mathbf{R}$ & $\mathbf{R}$ \\
\hline 144 & XXIX & $\mathbf{R}$ & $\mathbf{R}$ & $\mathbf{R}$ & $\mathbf{R}$ & $\mathbf{R}$ & $\mathbf{S}$ & $\mathbf{R}$ & $\mathbf{R}$ & $\mathbf{S}$ & $\mathbf{R}$ & $\mathbf{R}$ & $\mathbf{R}$ & $\mathbf{R}$ & $\mathbf{S}$ \\
\hline 145 & XXIX & $\mathbf{R}$ & $\mathbf{R}$ & $\mathbf{R}$ & $\mathbf{R}$ & $\mathbf{R}$ & $\mathbf{R}$ & $\mathbf{S}$ & $\mathbf{R}$ & $\mathbf{R}$ & $\mathbf{R}$ & $\mathbf{R}$ & $\mathbf{R}$ & $\mathbf{R}$ & $\mathbf{R}$ \\
\hline 116 & XXIX & $\mathbf{R}$ & $\mathbf{R}$ & $\mathbf{R}$ & $\mathbf{R}$ & $\mathbf{R}$ & $\mathbf{R}$ & $\mathbf{S}$ & $\mathbf{R}$ & $\mathbf{R}$ & $\mathbf{R}$ & $\mathbf{R}$ & $\mathbf{R}$ & $\mathbf{R}$ & $\mathbf{R}$ \\
\hline 3 & XXIX-b & $\mathbf{R}$ & $\mathbf{R}$ & $\mathbf{R}$ & $\mathbf{R}$ & $\mathbf{R}$ & $\mathbf{R}$ & $\mathbf{S}$ & $\mathbf{R}$ & $\mathbf{R}$ & $\mathbf{R}$ & $\mathbf{R}$ & $\mathbf{R}$ & $\mathbf{R}$ & $\mathbf{R}$ \\
\hline 8 & XXIX-b & $\mathbf{R}$ & $\mathbf{R}$ & $\mathbf{R}$ & $\mathbf{R}$ & $\mathbf{R}$ & $\mathbf{R}$ & I & $\mathbf{R}$ & $\mathbf{R}$ & $\mathbf{R}$ & $\mathbf{R}$ & $\mathbf{R}$ & $\mathbf{R}$ & $\mathbf{R}$ \\
\hline 69 & XXV & $\mathbf{R}$ & $\mathbf{R}$ & $\mathbf{R}$ & $\mathbf{R}$ & $\mathbf{R}$ & $\mathbf{S}$ & $\mathbf{S}$ & $\mathbf{R}$ & $\mathbf{I}$ & $\mathbf{S}$ & $\mathbf{R}$ & $\mathbf{S}$ & $\mathbf{R}$ & I \\
\hline 74 & XXV & $\mathbf{R}$ & $\mathbf{R}$ & $\mathbf{R}$ & $\mathbf{R}$ & $\mathbf{R}$ & $\mathbf{S}$ & $\mathbf{S}$ & $\mathbf{R}$ & $\mathbf{I}$ & $\mathbf{S}$ & $\mathbf{R}$ & $\mathbf{S}$ & $\mathbf{R}$ & $\mathbf{R}$ \\
\hline 77 & XXV & $\mathbf{R}$ & $\mathbf{R}$ & $\mathbf{R}$ & $\mathbf{R}$ & $\mathbf{R}$ & $\mathbf{S}$ & $\mathbf{S}$ & $\mathbf{R}$ & I & $\mathbf{S}$ & $\mathbf{R}$ & $\mathbf{S}$ & $\mathbf{R}$ & $\mathbf{R}$ \\
\hline 94 & XXV & $\mathbf{R}$ & $\mathbf{R}$ & $\mathbf{R}$ & $\mathbf{R}$ & $\mathbf{R}$ & $\mathbf{S}$ & I & $\mathbf{R}$ & $\mathbf{R}$ & $\mathbf{S}$ & $\mathbf{R}$ & $\mathbf{S}$ & $\mathbf{R}$ & I \\
\hline 105 & XXV & $\mathbf{R}$ & $\mathbf{R}$ & $\mathbf{R}$ & $\mathbf{R}$ & $\mathbf{R}$ & $\mathbf{S}$ & $\mathbf{S}$ & $\mathbf{R}$ & $\mathbf{R}$ & $\mathbf{S}$ & $\mathbf{R}$ & $\mathbf{S}$ & $\mathbf{R}$ & $\mathbf{S}$ \\
\hline 124 & XXV & $\mathbf{R}$ & $\mathbf{R}$ & $\mathbf{R}$ & $\mathbf{R}$ & $\mathbf{R}$ & $\mathbf{S}$ & $\mathbf{R}$ & $\mathbf{R}$ & $\mathbf{R}$ & $\mathbf{S}$ & $\mathbf{R}$ & $\mathbf{S}$ & $\mathbf{R}$ & $\mathbf{S}$ \\
\hline 135 & XXV & $\mathbf{R}$ & I & $\mathbf{R}$ & $\mathbf{R}$ & $\mathbf{R}$ & $\mathbf{S}$ & $\mathbf{S}$ & $\mathbf{S}$ & $\mathbf{S}$ & $\mathbf{R}$ & $\mathbf{R}$ & $\mathbf{R}$ & $\mathbf{R}$ & $\mathbf{R}$ \\
\hline 141 & XXV & $\mathbf{R}$ & $\mathbf{R}$ & $\mathbf{R}$ & $\mathbf{R}$ & $\mathbf{R}$ & $\mathbf{S}$ & $\mathbf{S}$ & $\mathbf{R}$ & $\mathbf{R}$ & $\mathbf{S}$ & $\mathbf{R}$ & $\mathbf{S}$ & $\mathbf{R}$ & $\mathbf{S}$ \\
\hline 104 & XXV-a & $\mathbf{R}$ & $\mathbf{R}$ & $\mathbf{R}$ & $\mathbf{R}$ & $\mathbf{R}$ & $\mathbf{S}$ & $\mathbf{S}$ & $\mathbf{R}$ & $\mathbf{S}$ & $\mathbf{S}$ & $\mathbf{R}$ & $\mathbf{S}$ & $\mathbf{R}$ & $\mathbf{S}$ \\
\hline 41 & XXXXIV & $\mathbf{R}$ & $\mathbf{R}$ & I & I & $\mathbf{R}$ & $\mathbf{S}$ & $\mathbf{S}$ & $\mathbf{S}$ & $\mathbf{S}$ & $\mathbf{S}$ & $\mathbf{R}$ & $\mathbf{S}$ & $\mathbf{R}$ & I \\
\hline 42 & XXXXIV & $\mathbf{R}$ & I & I & I & $\mathbf{R}$ & $\mathbf{S}$ & $\mathbf{S}$ & $\mathbf{R}$ & $\mathbf{S}$ & $\mathbf{S}$ & $\mathbf{R}$ & $\mathbf{S}$ & $\mathbf{R}$ & I \\
\hline 48 & XXXXIV & $\mathbf{R}$ & I & I & I & $\mathbf{R}$ & $\mathbf{S}$ & $\mathbf{S}$ & $\mathbf{R}$ & $\mathbf{S}$ & $\mathbf{S}$ & $\mathbf{R}$ & $\mathbf{S}$ & $\mathbf{R}$ & I \\
\hline 59 & XXXXIV & $\mathbf{R}$ & $\mathbf{R}$ & I & I & $\mathbf{R}$ & $\mathbf{S}$ & $\mathbf{S}$ & $\mathbf{R}$ & $\mathbf{R}$ & $\mathbf{S}$ & $\mathbf{R}$ & $\mathbf{S}$ & $\mathbf{R}$ & $\mathbf{R}$ \\
\hline 62 & XXXXIV & $\mathbf{R}$ & $\mathbf{R}$ & $\mathbf{R}$ & $\mathbf{R}$ & $\mathbf{R}$ & $\mathbf{S}$ & $\mathbf{S}$ & $\mathbf{R}$ & $\mathbf{R}$ & $\mathbf{S}$ & $\mathbf{R}$ & $\mathbf{R}$ & $\mathbf{R}$ & I \\
\hline 49 & $\underset{\mathbf{a}}{\text { XXXXIV- }}$ & $\mathbf{R}$ & I & I & I & $\mathbf{R}$ & $\mathbf{S}$ & $\mathbf{S}$ & $\mathbf{R}$ & $\mathbf{S}$ & $\mathbf{S}$ & $\mathbf{R}$ & $\mathbf{S}$ & $\mathbf{R}$ & $\mathbf{S}$ \\
\hline 44 & XXXXV & $\mathbf{R}$ & I & $\mathbf{R}$ & $\mathbf{R}$ & $\mathbf{R}$ & $\mathbf{S}$ & $\mathbf{S}$ & $\mathbf{R}$ & $\mathbf{S}$ & $\mathbf{S}$ & $\mathbf{R}$ & $\mathbf{S}$ & $\mathbf{R}$ & $\mathbf{R}$ \\
\hline 46 & $\begin{array}{c}\text { XXXXV - } \\
\text { b }\end{array}$ & $\mathbf{R}$ & I & $\mathbf{R}$ & $\mathbf{R}$ & $\mathbf{R}$ & $\mathbf{S}$ & $\mathbf{S}$ & $\mathbf{S}$ & $\mathbf{S}$ & $\mathbf{S}$ & $\mathbf{R}$ & $\mathbf{R}$ & I & $\mathbf{S}$ \\
\hline
\end{tabular}

Four of 135 strains could not be classified with PFGE. The cultures of these four resultant bacteria were checked for purity and these strains were confirmed as A. baumannii again. The same PFGE protocol was applied 3 times with markers but failed to obtain a result. The dendrogram of the 131 Acinetobacter spp. isolates is shown in Figure 1 and the antibiotic susceptibility profiles of the bacteria in the same cluster are given in Table 1. Also, PFGE results of Acinetobacter species are given in Figure 2. 


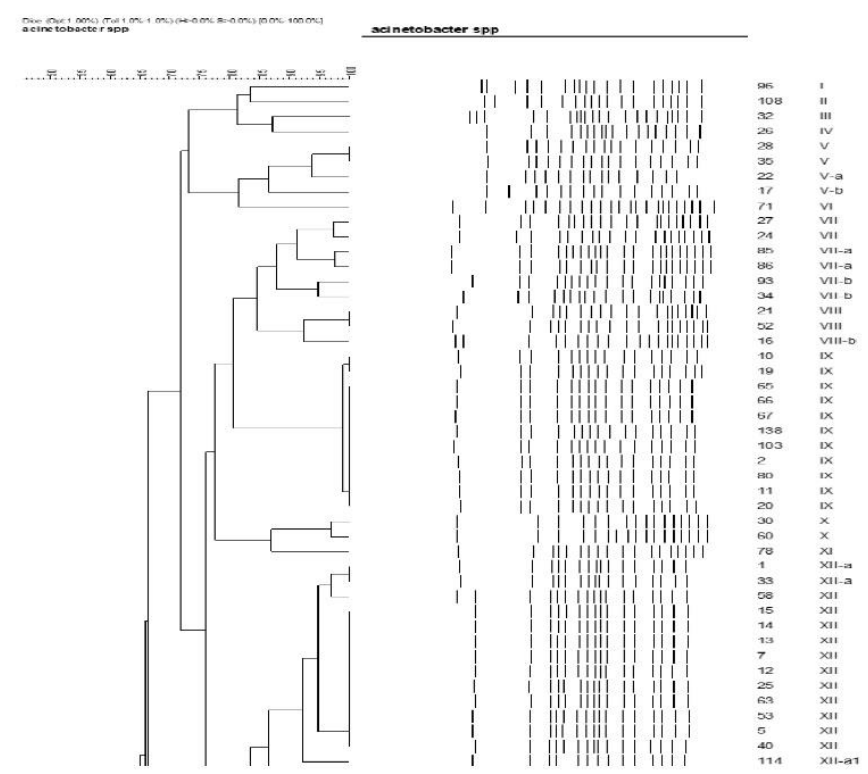

Fig. 1a. The dendogram of Acinetobacter strains

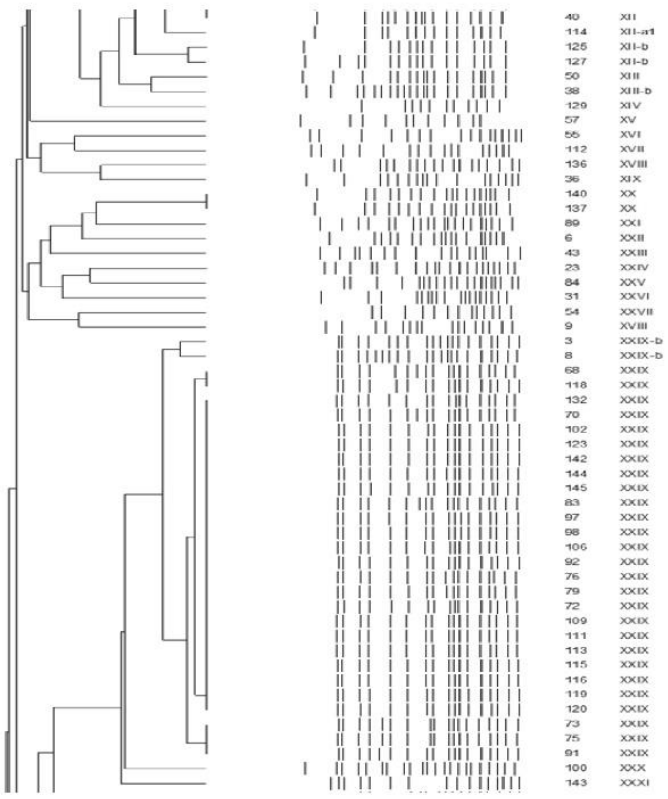

Fig. 1b. Continued. The dendogram of Acinetobacter strains 

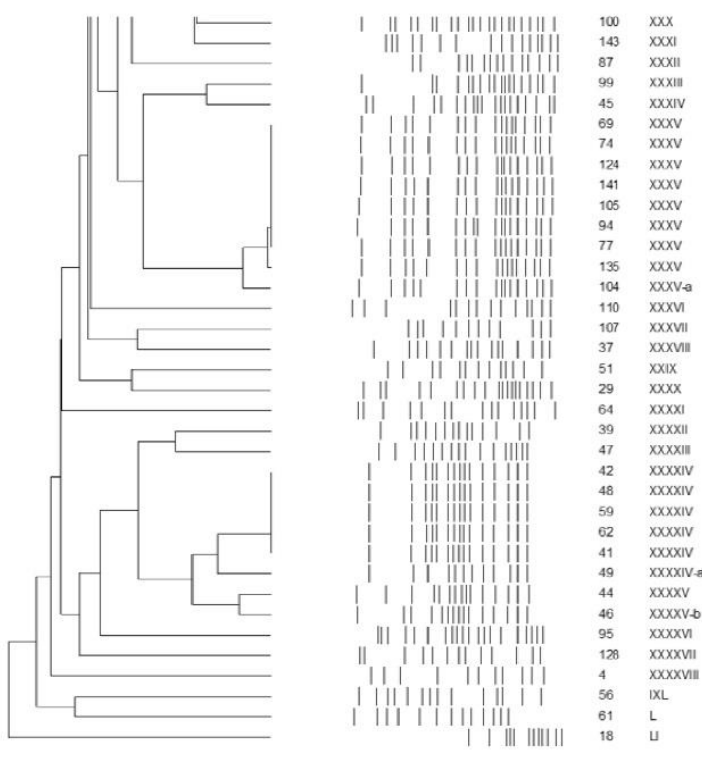

Figure1c. Continued. The dendogram of Acinetobacter strains

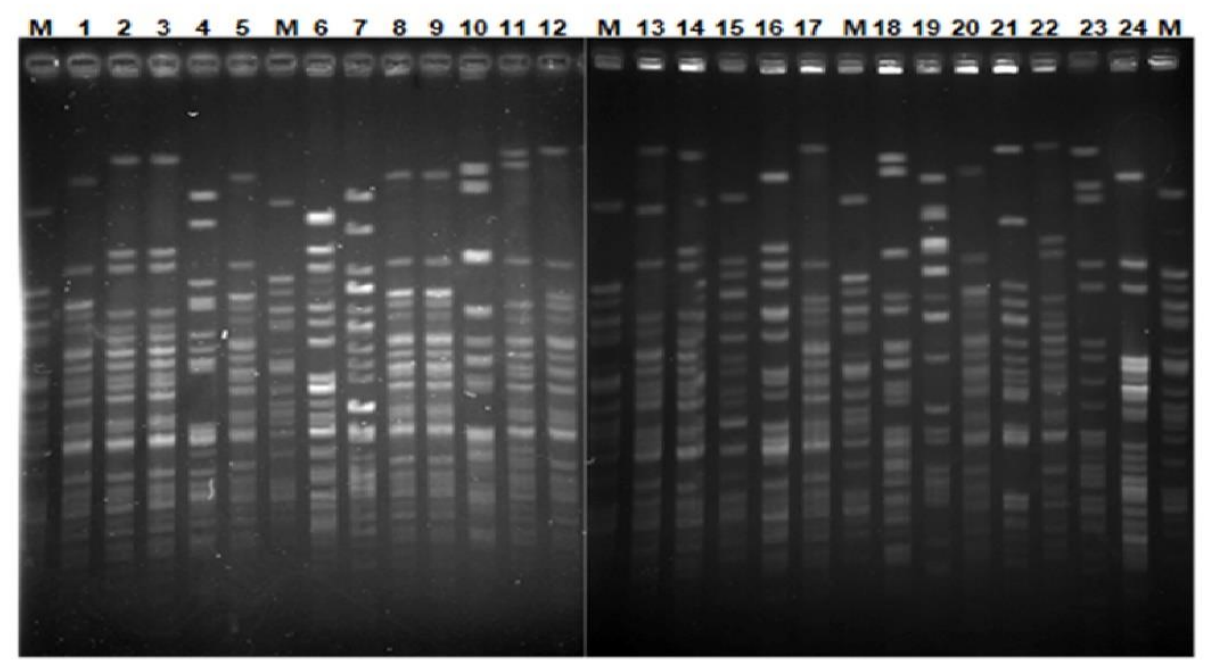

Fig. 2. PFGE results of Acinetobacter species

Of the 131 Acinetobacter spp. strains, 82 $(62.6 \%)$ were in clusters. These strains are located in 15 clusters. The number of strains varies between $2-27$ in the cluster. Two of the fifteen clusters (VII$a$ and XII-a) were also close to another two clusters and three (VII-b, XII-b, XXIX-b) were the possibly related with the other three clusters. Five of the strains were closely related and seven were related. According to these data, $72.3 \%$ of the strains were clonally related. The isolates of 37 strains $(28 \%)$ showed a specific PFGE profile. In total, 64 (48.9\%) PFGE patterns were determined for 131 strains. 
The maximum number of strains in a cluster (27 strains) were in the twelfth cluster. The longest surviving clone was in the sixth cluster and survived twenty-seven months. The shortest period was in the eleventh cluster with sixteen days.

Strains 111 and 123, typed by PFGE, belonged to the same patient. Both strains are in the twelfth cluster. The first strain from the patient was isolated on 30.7.2007 in the paracentesis fluid culture of the patient who was hospitalized with diagnosis of liver failure and diabetes. The second strain was isolated on a blood culture sample after transplantation on October 15, 2007.

Strains 63 and 70, which were typed by PFGE, belonged to the same patient. The first strain was isolated on 12.1.2007 in the urine culture of the patient hospitalized in the neurosurgical intensive care unit. The strain is in the eighth cluster. The second strain was isolated in a blood culture sample three months later. The strain is located in the twelfth cluster with a different set.

Strains 28 and 29 were isolated from the same patient at an eight-month interval. Although the 28th isolate was in the first cluster, strain 29 was not included in any cluster.

Strains 47 and 48 were isolated in the blood and tracheal aspiration culture samples of a patient at a one-week interval. The first strain was not included in any cluster. The second strain was in the fifteenth cluster.

Strains 82 and 139, which were typed by PFGE, were isolated from the same patient at seven-month intervals. Both strains could not be identified by the PFGE method.

Four of the 130 patients included in the study had liver transplantation. The strains isolated from these four patients are located in the twelfth cluster and belong to patients who were hospitalized in the organ transplantation and anesthesia intensive care unit.

Twenty-five of the 27 strains were resistant to imipenem and amikacin in the twelfth cluster. Compared to other clusters, the most resistance to antibiotics was observed in this cluster.

\section{Discussion}

In this study, the number of strains resistant to three or more groups of antibiotics (MDR) number was $77(57 \%)$. XDR strains and pandrug resistant strains numbered 23 (17\%) and 2 (1.5\%), respectively. Also, 34 Acinetobacter spp. had colistin and tigecycline sensitivities evaluated by using the disk diffusion method. Two strains were found to be resistant to colistin, while nine were resistant to tigecycline.

In the literature, the resistance profiles of $402 \mathrm{~A}$. baumannii strains were compared. This profile has statistically significantly increased for all tested antibiotics compared to the previous year (Gazi et al., 2005).

Gülhan et al. (2007) monitored the resistance changes from 2004 to 2006. They found a statistically significant increase in carbapenem resistance from $7 \%$ to $25 \%$ and for ciprofloxacin from $54 \%$ to $82 \%$.

In a study comprising 1532 clinical isolates over a 6-year period (1991 to 1996), resistance rates increased for ciprofloxacin from $54.4 \%$ to $90.4 \%$, for amikacin from $21 \%$ to $83.7 \%$, for trimethoprim / sulfamethoxazole from $41.1 \%$ to $88.9 \%$, for imipenem from $1.3 \%$ to $80 \%$, and for ampicillin / sulbactam from $65.7 \%$ to $84.1 \%$ in Spain (Ruiz et al., 1999).

Infections caused by MDR Pseudomonas aeruginosa, A. baumannii and Klebsiella pneumoniae strains have become a common problem in health institutions. These strains have consistently developed resistance to antibiotics, leading to the emergence of PDR isolates that are susceptible to only one antimicrobial agent and are resistant to all available drugs. The frequency of PDR clinical isolates, the treatment options of the infections associated with these isolates, and the mortality and morbidity rates are of great importance in terms of clinical and public health (Falagas and Bliziotis, 2007).

Gales et al. (2006) detected a polymyxin resistance rate of $2 \%$ and PDR rate of $0.3 \%$ in 2621 Acinetobacter spp. isolates in a surveillance study between 2001 and 2004. Henwood and colleagues (2002) identified the polymyxin resistance rate as $44 \%$ among $443 \mathrm{~A}$. baumannii isolates in a surveillance study involving 25 laboratories.

In a surveillance study conducted in hospitals in ten different geographic regions, it was shown that imipenem resistance increased to $18.2 \%$ in 2004 from $4.5 \%$ in 2003 (Catchpole et al., 1997). In a study conducted in a 1600-bed tertiary education hospital in Beijing, imipenem resistance was $5 \%$ in 1993 to 2003 and increased rapidly to $50 \%$ in intensive care units and to $20 \%$ in non-intensive care units in 2004.

While imipenem resistance was not found in our hospital in 2005, it increased to $22 \%$ in 2006 and to $39 \%$ in 2007. These isolates were also MDR Acinetobacter isolates. In our hospital, carbapenem treatment is begun prophylactically and empirically 
for patients who are thought to have infection due to Gram negative bacteria. This contributes to the increase in carbapenem resistance among Acinetobacter species.

In a study, a clone survived for about 6 years in a hospital (Wang et al., 2007). Prashanth et al. (2005) identified 71 Acinetobacter spp. isolated in intensive care units using PFGE, and 59 (83\%) were determined to have different patterns.

The reasons for different antibiotic patterns of isolates in the same clones can be explained in several ways. The first is that the genetic event that leads to resistance development in the strains is different for the resistance profile and is different than the rate of genetic change that causes the PFGE profile although they are clonally related. The second reason is that the source of resistance in the strains exhibiting a common resistance profile, may be in the form of plasmid transfer, although they are different clonally. It is generally accepted that mutual resistance and PFGE profile is parallel to the clonal spread in strains showing common resistance (Maslow et al., 1993; Falagas and Kopterides, 2006).

In a similar study, 36 PFGE patterns were present in $66 \mathrm{~A}$. baumannii isolates studied and their genotype analysis found twelve clusters. They found the epidemiological relationship rate to be $80.3 \%$ (Çetin et al., 2009).

In our hospital, all intensive care wards are on the same floor and hospital staff and ventilator devices are used together. Patient transfers are made frequently between intensive care units. This increases the spread of $A$. baumannii clones in our hospital. In addition, 35 isolates were produced from the cultures of respiratory tract samples and patients were connected to the ventilator device. This is compatible with the literature which described that "the biggest risk factor for $A$. baumannii infections was mechanical ventilation" (Villari et al., 1999).

In our study, the antibiotic resistance profiles of the strains belonging to the same cluster were substantially similar and it was shown that antibiotic profiles in the same cluster could be different. Additionally, strains isolated from the same patient may belong to the same cluster or belong to a different cluster.

Hui Wang et al. (2007) identified 221 imipenem resistant isolates and 15 patterns that contain two or more subtypes in 11 hospitals between 1999 and 2005 with PFGE. During this period, they found clonal extension in 10 hospitals in four cities. Approximate mortality rates were $22.1 \%$ and
$40.2 \%$, respectively. Twenty-eight cases were classified as colonized. The development time of the infection was 27.8 days. All patients had an underlying disease and $70 \%$ used broad-spectrum antibiotics. It was also found that a clone maintained its existence in the hospital for 6 years. However, no patient transfers were found between hospitals with clonal extension.

In our study, the PFGE analysis of 34 (26\%) isolates that had imipenem resistance consisted of fifteen patterns and two clusters. The first cluster with imipenem resistance consists of twenty-five isolates. The second cluster consists of two isolates. The first cluster is likely related to the second cluster. The clone survived in our hospital for about nine months. There was clonal invasion in the ten services. The propagation and transmission are probably associated with patient transfer between wards, shared hospital staff, and common use of ventilator devices. Twenty-two of the isolates were produced in the intensive care units and twelve from other wards. Fifteen isolates were found in lower respiratory tract culture samples, nine in blood culture samples, four in urine culture samples, and three isolates in paracentesis and wound culture samples. The mortality rate (47\%) for this clone was found to be significantly higher than the average found in the hospital (36\%).

During the three years of this study, $A$. baumannii-related hospital infections were encountered, especially in intensive care units. Acinetobacter strains produced as a hospital agent have high antibiotic resistance and resistance increases significantly over the years. The increase in MDR, XDR and PDR rates among the strains is noteworthy. It was determined that the degree of transmission of the strains was quite high among the patients, the clones in the hospital were able to stay in the environment for long periods and the general patient mortality rates were higher than the other studies. The necessity of ensuring more effective protection and control measures in our hospital was shown with the support of molecular typing studies.

\section{Acknowledgments}

This study was produced from the specialist thesis of Ahmet Caliskan and includes part of project SBAG 106S211 supported by TUBITAK. 
Ethics Committee Approval: Ethics committee approval was received for this study from Clinical Research Ethics Committee of Inonu University. Ethics no: 2008/003

Peer-review: Externally peer-reviewed.

Author Contributions: Concept- A.Ç, R.D.; Design A.Ç, R.D.; Supervision- A.Ç, R.D.; Materials A.Ç., R.D, C.A.G.; Data Collection and/or Processing A.Ç, R.D, C.A.G.; Analysis and/or Interpretation-A.C,， R, R.D. C.A.G. ; Literature Review- A.Ç, N.I.; Writing-A.Ç, R.D, N.I.; Critical Review- A.Ç, R.D, C.A.G, N.I.

Conflict of Interest: No conflict of interest was declared by the author.

Financial Disclosure: This study has been financed by TUBITAK (Project number: SBAG 106S211).

\section{References}

Aber CR, Mackel DC. Epidemiologic typing of nosocomial microorganisms. The American Journal of Medicine. 1981;70:898-905.

Aparajita Richard V, Goering Shabbir S, Foley SL, Zervos M. Application of Molecular Techniques to the Study of Hospital Infection. Clinical Microbiology. 2006;19:512- 530.

Arbeit RD. Laboratory procedures for the epidemiologic analysis of microorganisms 1995., p. 190-208. In Murray PR, Baron EJ, Pfaller MA, Tenover FC, Yolken RH (ed.), Manual of clinical microbiology, 6th ed. American Society for Microbiology, Washington, D.C.

Beck-Sague CM, Jarvis WR, Brook JH, Culver DH, Potts A, Gay A et al. Epidemic bacteremia due to Acinetobacter baumannii in five intensive care unit. American Journal of Epidemiology. 1990;132:723-733.

Bergogne-Berezin E, Towner KJ. Acinetobacter spp.as nosocomial pathogens: microbiological, clinical, and epidemilogical features. Clinical Microbiology Review. 1996;9:148-165.

Bou G, Cervero G, Dominguez MA, Querada C, Martínez-Beltrána J. PCR-based DNA fingerprinting(REP-PCR, AP-PCR) and pulsedfield gel electrophoresis characterization of a nosocomial outbreak caused by imipenem and meropenem- resistant Acinetobacter baumannii. Clinical Microbiology and Infection. 2000;6:635-643.
Buxton AE, Anderson RL, Werdegar D, Ernest Atlas MD. Nosocomial respiratory tract infection and colonization with Acinetobacter calcoaceticus. The American Journal of Medicine. 1978;65:507-513.

Catchpole CR, Andrews JM, Brenwald N, Wise R. A reassessment of the in-vitro activity of colistin sulphomethate sodium. Journal of Antimicrobial Chemotheraphy. 1997;39:255-60.

Clinical and Laboratory Standards Institute 2008. Performance standart for antimicrobial disk susceptibility Testing; Approved standard M2A9 and M7-A7, 18 th ed. CLSI, Wayne, PA.

Corbella X, Pujol M, Ayast J, Sendra M, Ardanury C, Dominguez MA, Linares J, Ariza J, Gudiol F. Relevance of digestive tract colonization in the epidemiology of nasocomial infections due to multi resistant Acinetobacter baumannii. Clinical Microbiology and Infectious Disease. 1996;23:329-334.

Durmaz R, Otlu B, Calıskan A, Gursoy N. A Rapid Pulsed-field Gel Electrophoresis (PFGE) Protocol Developed for Subtyping Acinetobacter baumannii, Escherichia coli, and Klebsiella spp. ANKEM. 2007;21(2):113-117

Cetin Sesli E, Durmaz R, Tetik T, Otlu B, Kaya S, Caliskan A. Epidemiologic characterization of nosocomial Acinetobacter baumannii infections in a Turkish university hospital by pulsed-field gel electrophoresis. American Journal of Infection Control. 2009;37: 56-64.

Falagas ME, Bliziotis IA, Kasiakou SK, Samonis G. Outcome of infections due to pandrug-resistance gram-negative bacteria. BMC Infectious Disease 2005; 5: 24.

Falagas ME, Bliziotis IA. Pandrug-resistant Gramnegative bacteria: the dawn of the post-antibiotic era? International Journal of Antimicrobial Agents 2007;29: 630-636

Falagas ME, Kopterides P. Risk factors for the isolation of multi-drug-resistant Acinetobacter baumannii and Pseudomonas aeruginosa: a systematic review of the literature. Journal of Hospital Infection. 2006; 64: 7-15.

Gales AC, Jones RN, Sader HS. Global assessment of the antimicrobial activity of polymyxin B against 54731 clinical isolates of Gram negative bacilli: report from the SENTRY antimicrobial surveillance programme (2001-2004). Clinical Microbiology Infection. 2006;12:315-21. 
Gazi H, Sürücüoglu S, Kurutepe S, Inmez E, Dinc G, Özbakkaloğlu B. In vitro Antibiotic Susceptibilities in Nosocomial Acinetobacter baumannii Strains Isolated from Intensive Care Unit and other Clinics. ANKEM. 2005;19(3):115-118.

Giske CG, Monnet DL, Cars O, Carmeli Y. On behalf of ReAct-Action on Antibiotic Resistance. Antimicrobial Agents and Chemotheraphy. 2008; 52: 813-21

Goering RV. Molecular epidemiology of nosocomial infection: analysis of chromosomal restriction fragment patterns by pulsed-field gel electrophoresis. Infection Control and Hospital Epidemiology. 1993;14:595-600.

Gulhan B, Ozekinci T, Atmaca S, Bilek H. Antibiotic Resistance of Acinetobacter baumannii Strains Isolated in 2004-2006 Years. ANKEM. 2007;21(1):32-36.

Henwood CJ, Gatward T,Warner M, James D, Stockdale MW, Spence RP, et al. Antibiotic resistance among clinical isolates of Acinetobacter in the UK, and in vitro evaluation of tigecycline (GAR-936). Journal of Antimicrobial Chemotheraphy. 2002;49:47987.

Hsueh PR, LJ, Chen CY, Chen WE, Ho SW, Luh KT. Pandrug-resistant Acinetobacter baumannii causing nasocomial infections in a university hospital, Taiwan. Emerging Infectious Disease. 2002;8:827-832.

Jain R, Danziger LH. Multidrug-resistant Acinetobacter infections: an emerging challenge to clinicians. Annals of Pharmacotherapy. 2004;38:1449-1459.

Jarvis WR. Usefulness of molecular epidemiology for outbreak investigations. Infection Control \& Hospital Epidemiology. 1994;15:500-503

Jones R, Feraro M, Relle L, Schreckenbeger P, Swenson J, Sader H. Multicenter studies of tigecycline Disk Diffusion Susceptibity Results for Acinetobacter spp. Journal of Clinical Microbiology. 2007;6.227-230.

Li J, Nation RL, Milne RW, Turnidge JD, Coulthard K. Evaluation of colistin as an agent against multi-resistant gram-negative bacteria. International Journal of Antimicrobial Agents 2005;25:11-25.

Lortholary O, Fagon JY, Hoi AB, Slama MA, Pierre J, Giral P, Rosenzweig R, Gutmann L, Safar M, Acar J. Nosocomial acquisition of multiresistant Acinetobacter baumannii: risk factors and prognosis. Clinical Infectious Disease. 1995, 20:790-796.
Lyons RW. Ecology, clinical significance and antimicrobial susceptibilty of Acinetobacter and Moraxella. In: Gilardi GL. Ed. Nonfermantative Gram Negative Rods: Laboratory Identification and Clinical Aspects: New York: Marcel dekker, 1985;159-179.

Maslow JN, Slutsky AM, Arbeit RD. Application of pulsed-field gel electrophoresis to molecular epidemiology. In: Persing HD, Smith TF, Tenover FC, White TJ, eds. Diagnostic molecular microbiology: principles and applications. Washington, DC: American Society for Microbiology; 1993:563-72.

Mulin B, Talon D, Viel JF, Vincent C, Leprat R, Thouverez M, et al. Risk factors for nosocomial colonization with multiresistant Acinetobacter baumannii, European Journal of Clinical Microbiology and Infectious Diseases. 1995; 14(7):569-76.

Parvez FM, Jarvis WR. Nosocomial infections in the nursery. Seminar in Pediatric Infectious Disease. 1999;10:119-29.

Prashanth K, Badrinath S. Epidemiological investigation of nosocomial Acinetobacter infections using arbitrarily primed PCR \& pulse field gel electrophoresis. Indian Journal of Medical Research. 2005:11;122:408-418

Ruiz J, Nunez ML, Perez J, Simarro E, MartinezCampos L, Gomez J. Evolution of resistance among clinical isolates of Acinetobacter over a 6-year period, European Journal of Clinical Microbiology and Infectious Disease. 1999;18(4):292-5.

Seifert H, Baginski R, Schulze A, Pulverer G. Antimicrobial susceptibility of Acinetobacter species. Zentralblatt fur Bakteriologie: International Journal of Medical Microbiology. 1993;37:750-753.

Siegman-Igra Y, Bar-Yosef S, Gorea A, Avram J. Nosocomial Acinetobacter meningitis secondary to invasive procedues: report of 25 cases and review. Clinical Infectious Disease. 1993; 17:843-849.

Tenover FC, Arbeit RD, Goering RV. How to select and interpret molecular strain typing methods for epidemiological studies of bacterial infections: a review for healthcare epidemiologists. Molecular Typing Working Group of the Society for Healthcare Epidemiology of America. Infection Control and Hospital Epidemiology. 1997;18(6):426-39. 
Villari P, Iacuzio L, Vozzella EA, Bosco U. 1999. Unusual genetic heterogeneity of Acinetobacter baumanni isolates in a university hospital in Italy. The American Journal of Infection Control. 27: 247-253.

Villegas MV, Hartstein Al. Acinetobacter outbreaks,1977-2000. Infection Control and Hospital Epidemiology. 2003;24:284-295.

Wang H, Guo P, Sun H, Wang, H, Yang O, Chen, $\mathrm{M}$, et al. Molecular Epidemiology of Clinical Isolates of Carbapenem-Resistant Acinetobacter spp. from Chinese Hospitals. Antimicrobial Agents and Chemotherapy. 2007; 10: 4022-4028

Webster CA, Crove M, Humphreys H, Towner KJ. Surveillance of and adult intensive care unit for long-term persistence of a multi-resistant strain of Acinetobacter baumannii. Journal of Clinical Microbiology. 1998;17:171-176. 